Volume 136, Number 1, January 2008, Pages 351-358

S 0002-9939(07)09062-4

Article electronically published on September 25, 2007

\title{
ON LOCAL SOLVABILITY OF CERTAIN DIFFERENTIAL COMPLEXES
}

\author{
FABIO NICOLA
}

(Communicated by David S. Tartakoff)

\begin{abstract}
In any locally integrable structure a differential complex induced by the de Rham differential is naturally defined. We give necessary conditions, in terms of the signature of the Levi form, for its local solvability with a prescribed rate of shrinking.
\end{abstract}

\section{Introduction AND STATEMENT OF THE RESUlts}

The present paper deals with some invariants associated with locally integrable structures, recently introduced by C.D. Hill and M. Nacinovich [10] in the context of $C R$ manifolds.

We recall (see Treves [22] for details) that a locally integrable structure on a manifold $M$ is defined by a bundle $\mathcal{V} \subset \mathbb{C} T M$ satisfying the Frobenius condition and such that the subbundle $T^{\prime} \subset \mathbb{C} T^{*} M$ orthogonal to $\mathcal{V}$ is locally spanned by exact differentials. As usual we will denote by $T^{0}=T^{\prime} \cap T^{*} M$ the so-called characteristic set. For any open subset $\Omega \subset M$ the space of $(p, q)$-forms $C^{\infty}\left(\Omega, \Lambda^{p, q}\right)$ is then defined and the de Rham differential induces a map

$$
\begin{aligned}
\mathrm{d}^{\prime}: & C^{\infty}\left(\Omega, \Lambda^{p, q}\right) & \rightarrow C^{\infty}\left(\Omega, \Lambda^{p, q+1}\right), \\
\mathrm{d}^{\prime}: & \mathcal{D}^{\prime}\left(\Omega, \Lambda^{p, q}\right) & \rightarrow \mathcal{D}^{\prime}\left(\Omega, \Lambda^{p, q+1}\right) .
\end{aligned}
$$

When $\mathcal{V} \cap \overline{\mathcal{V}}=0$ the structure is called $C R$ and $\mathrm{d}^{\prime}$ is the tangential Cauchy-Riemann operator.

Now let $\mathbf{g}$ be a Riemannian metric near a given point $x_{0} \in M$, and let $B\left(x_{0}, r\right)$ be the corresponding open ball of radius $r>0$. We are concerned with the following local solvability property that involves two real numbers $r \geq r^{\prime}>0(1 \leq q \leq$ $\operatorname{rank} \mathcal{V})$ :

For any given cocycle $f \in C^{\infty}\left(B\left(x_{0}, r\right), \Lambda^{0, q}\right)$ there exists a distribution

$$
\text { section } u \in \mathcal{D}^{\prime}\left(B\left(x_{0}, r^{\prime}\right), \Lambda^{0, q-1}\right) \text { solving } \mathrm{d}^{\prime} u=f \text { in } B\left(x_{0}, r^{\prime}\right) \text {. }
$$

Following [10] we define ${ }^{1}$ the functions

$$
\kappa_{q, x_{0}}^{\mathrm{g}}(r)=\sup \left\{r^{\prime}>0:(1.1) \text { is valid }\right\},
$$

Received by the editors November 15, 2005 and, in revised form, June 7, 2006.

2000 Mathematics Subject Classification. Primary 58J10; Secondary 35N05.

${ }^{1}$ With the convention that $\sup \emptyset=0$ and $\log 0=-\infty$. 
and

$$
\nu_{q}^{-}\left(x_{0}\right)=\liminf _{r \searrow 0} \frac{\log \kappa_{q, x_{0}}^{\mathbf{g}}(r)}{\log r} \text { and } \nu_{q}^{+}\left(x_{0}\right)=\limsup _{r \searrow 0} \frac{\log \kappa_{q, x_{0}}^{\mathbf{g}}(r)}{\log r} .
$$

The functions $\nu_{q}^{ \pm}$take values in $[1,+\infty]$ and, as is shown in [10], are in fact independent of the choice of the Riemannian metric g. We refer to Section 7 of [10] for geometric-cohomological interpretations of these invariants, which apply to our context too. In any case, here we do not use results proved in [10].

We now present our result. To this end, we recall that at any point $\left(x_{0}, \omega_{0}\right) \in T^{0}$ a sesquilinear form $\mathcal{B}_{\left(x_{0}, \omega_{0}\right)}: \mathcal{V}_{x_{0}} \times \mathcal{V}_{x_{0}} \rightarrow \mathbb{C}\left(\mathcal{V}_{x_{0}}\right.$ is the fibre above $\left.x_{0}\right)$ is well defined by

$$
\mathcal{B}_{\left(x_{0}, \omega_{0}\right)}\left(\mathbf{v}_{1}, \mathbf{v}_{2}\right)=\left\langle\omega_{0},\left.(2 \iota)^{-1}\left[V_{1}, \overline{V_{2}}\right]\right|_{x_{0}}\right\rangle,
$$

with $\mathbf{v}_{1}, \mathbf{v}_{2} \in \mathcal{V}_{x_{0}}$, where $V_{1}$ and $V_{2}$ are smooth sections of $\mathcal{V}$ such that $\left.V_{1}\right|_{x_{0}}=\mathbf{v}_{1}$, $\left.V_{2}\right|_{x_{0}}=\mathbf{v}_{2}$. The associated quadratic form $\mathcal{V}_{x_{0}} \ni \mathbf{v} \mapsto \mathcal{B}_{\left(x_{0}, \omega_{0}\right)}(\mathbf{v}, \mathbf{v})$, or $\mathcal{B}_{\left(x_{0}, \omega_{0}\right)}$ itself, is known as the Levi form.

Theorem 1.1. Let $\left(x_{0}, \omega_{0}\right) \in T^{0}, \omega_{0} \neq 0$. Suppose that $\mathcal{B}_{\left(x_{0}, \omega_{0}\right)}$ has exactly $q$ positive eigenvalues, $1 \leq q \leq \mathrm{rank} \mathcal{V}$, and that its restriction to $\mathcal{V}_{x_{0}} \cap \overline{\mathcal{V}}_{x_{0}}$ is nondegenerate. Then for every Riemannian metric $\mathbf{g}$ it turns out that

$$
\kappa_{q, x_{0}}^{\mathbf{g}}(r)=O\left(r^{3 / 2}\right), \quad \text { as } r \searrow 0 .
$$

In particular, $\nu_{q}^{-}\left(x_{0}\right) \geq 3 / 2$.

The meaning of Theorem 1.1 is that, under its assumptions, either the system in (1.1) is not locally solvable even after shrinking the neighborhood or, if it is, the solvability neighborhood has to be taken much smaller than the initial one. This generalizes Theorem 7.3 of [10] (there $\mathcal{V}_{x} \cap \overline{\mathcal{V}}_{x}=\{0\}$ for every $x \in M$ ).

Indeed, several papers are devoted to the interplay between the signature of the Levi form and the possibility of locally solving the system $\mathrm{d}^{\prime} u=f$, possibly in a smaller neighborhood; see Lewy [13], Hörmander [11], Andreotti and Hill [2], Andreotti, Fredricks, and Nacinovich [1], Nacinovich [15, 16, 17], Kashiwara and Schapira [12], Michel [14], Treves [20, 21, 22, 23], Chen and Shaw [9], Peloso and Ricci [19], Hill and Nacinovich [10], Nicola [18]. We also refer, for other invariants, to the contributions by Cordaro and Hounie [4, 5, 6], Cordaro and Treves [7, 8], Chanillo and Treves [3].

One of the new ideas in [10], which inspired this work, was the measurement of the rate of shrinking of the neighborhood in solving the system in (1.1). In this connection Theorem 1.1 above may be of interest when the Levi form degenerates $\left(\right.$ on $\mathcal{V}_{x_{0}}$ ). In fact, it is known from Theorem XVIII.3.1 of [22] that if one supposes, in addition, that the Levi form at $\left(x_{0}, \omega_{0}\right)$ is non-degenerate on $\mathcal{V}_{x_{0}}$, then the Poincaré lemma does not hold at $x_{0}$ in degree $q$ (with the expression "Poincaré lemma" we mean that for every $r>0$ there exists $0<r^{\prime} \leq r$ such that (1.1) is valid for any Riemannian metric $\mathbf{g}$, i.e. $\left.\kappa_{q, x_{0}}^{\mathrm{g}}(r)>0\right)$. This was a generalization of the classical result of $[1]$.

It is important to observe that, generally, under the assumptions of Theorem 1.1 the Poincaré lemma at $x_{0}$ in degree $q$ can hold or not; we refer to Nacinovich [16] for positive results, and to [10] for negative results.

An interesting question which remains open is an estimate of $\kappa_{q, x_{0}}^{\mathbf{g}}(r)$ as $r \searrow 0$ when the Levi form is allowed to degererate on $\mathcal{V}_{x_{0}} \cap \overline{\mathcal{V}}_{x_{0}}$ as well. In fact, it seems 
quite difficult in that case to give a general condition with an invariant meaning, although special situations can be treated with the techniques of [7] and [10].

This paper is organized as follows. Theorem 1.1 is proved in Section 3. Section 2 is devoted to some preliminary results. Eventually in Section 4 we discuss an example and the possibility of improving Theorem 1.1 in special cases.

\section{Preliminaries}

In this section we briefly discuss the notation used in this paper and some basic results in the theory of locally integrable structure; we refer to Treves [22] for proofs. In particular, we now recall the existence of special coordinates that will play a crucial role in the next section (see section I.9 of [22]).

We regard $x_{0}$ as the origin of the coordinates, hence it will be denoted by 0 . Let $n=\operatorname{dim}_{\mathbb{C}} \mathcal{V}_{0}, d=\operatorname{dim}_{\mathbb{R}} T_{0}^{0}, \nu=n-\operatorname{dim}_{\mathbb{C}}\left(\mathcal{V}_{0} \cap \overline{\mathcal{V}}_{0}\right)$

Proposition 2.1. Let $\left(0, \omega_{0}\right) \in T^{0}, \omega_{0} \neq 0$, and suppose that the restriction of the Levi form $\mathcal{B}_{\left(0, \omega_{0}\right)}$ to $\mathcal{V}_{0} \cap \overline{\mathcal{V}}_{0}$ is non-degenerate. There exist coordinates $x_{j}, y_{j}, s_{k}$ and $t_{l}, j=1, \ldots, \nu, k=1, \ldots, d, l=1, \ldots, n-\nu$, and smooth real functions $\phi_{k}(x, y, s, t), k=1, \ldots d$, in a neighborhood $\mathcal{O}$ of 0 , satisfying

$$
\left.\phi_{k}\right|_{0}=0 \text { and }\left.d \phi_{k}\right|_{0}=0 \text {, }
$$

such that

$$
\left\{\begin{array}{l}
z_{j}:=x_{j}+\iota y_{j}, j=1, \ldots, \nu, \\
w_{k}:=s_{k}+\iota \phi_{k}(x, y, s, t), k=1, \ldots, d,
\end{array}\right.
$$

define a system of first integrals for $\mathcal{V}$, i.e. their differential span $\left.T^{\prime}\right|_{\mathcal{O}}$. Moreover, with respect to the basis

$$
\left\{\left.\frac{\partial}{\partial \bar{z}_{j}}\right|_{0},\left.\frac{\partial}{\partial t_{l}}\right|_{0} ; j=1, \ldots, \nu, l=1, \ldots, n-\nu\right\}
$$

of $\mathcal{V}_{0}$, the Levi form $\mathcal{B}_{\left(0, \omega_{0}\right)}$ reads

$$
\sum_{j=1}^{p^{\prime \prime}}\left|\zeta_{j}\right|^{2}-\sum_{j=p^{\prime \prime}+1}^{p^{\prime \prime}+q^{\prime \prime}}\left|\zeta_{j}\right|^{2}+\sum_{l=1}^{p^{\prime}}\left|\tau_{l}\right|^{2}-\sum_{l=p^{\prime}+1}^{n-\nu}\left|\tau_{l}\right|^{2} .
$$

In particular

$$
\mathrm{d}^{\prime} z_{j}=0, \mathrm{~d}^{\prime} w_{k}=0, \quad j=1, \ldots, \nu, k=1, \ldots, d .
$$

In these coordinates we have $T_{0}^{0}=\operatorname{span}_{\mathbb{R}}\left\{\left.d s_{k}\right|_{0} ; k=1, \ldots, d\right\}$, so that $\omega_{0}=$ $\left.\sum_{k=1}^{d} \sigma_{k} d s_{k}\right|_{0}$, with $\sigma_{k} \in \mathbb{R}$. By (I.9.2) of [22] we have

$$
\mathcal{B}_{\left(0, \omega_{0}\right)}\left(\mathbf{v}_{1}, \mathbf{v}_{2}\right)=\left.\sum_{k=1}^{d} \sigma_{k}\left(V_{1} \bar{V}_{2} \phi_{k}\right)\right|_{0}
$$


with $V_{1}$ and $V_{2}$ smooth sections of $\mathcal{V}$ extending $\mathbf{v}_{1}$ and $\mathbf{v}_{2}$, respectively. Hence, upon setting $\Phi=\sum_{k=1}^{d} \sigma_{k} \phi_{k}$, we see from (2.2) that

$$
\begin{aligned}
\left.\Phi\right|_{s=0}= & \sum_{i, j=1}^{\nu} \frac{\partial^{2} \Phi}{\partial z_{i} \partial \bar{z}_{j}}(0) z_{i} \bar{z}_{j}+\frac{1}{2} \sum_{l, k=1}^{n-\nu} \frac{\partial^{2} \Phi}{\partial t_{l} \partial t_{k}}(0) t_{l} t_{k}+\operatorname{Re} \sum_{j=1}^{\nu} \sum_{l=1}^{k} \frac{\partial^{2} \Phi}{\partial \bar{z}_{j} \partial t_{l}}(0) \bar{z}_{j} t_{l} \\
& +\operatorname{Re} \sum_{i, j=1}^{\nu} \frac{\partial^{2} \Phi}{\partial z_{i} \partial z_{j}}(0) z_{i} z_{j}+O\left(|z|^{3}+|t|^{3}\right) \\
= & \sum_{j=1}^{p^{\prime \prime}}\left|z_{j}\right|^{2}-\sum_{j=p^{\prime \prime}+1}^{p^{\prime \prime}+q^{\prime \prime}}\left|z_{j}\right|^{2}+\frac{1}{2} \sum_{l=1}^{p^{\prime}} t_{l}^{2}-\frac{1}{2} \sum_{l=p^{\prime}+1}^{n-\nu} t_{l}^{2} \\
& +\operatorname{Re} \sum_{i, j=1}^{\nu} \frac{\partial^{2} \Phi}{\partial z_{i} \partial z_{j}}(0) z_{i} z_{j}+O\left(|z|^{3}+|t|^{3}\right) .
\end{aligned}
$$

We also observe that by (2.1) we have

$$
\Phi=\Phi_{s=0}+O(|s|(|z|+|s|+|t|))=\left.\Phi\right|_{s=0}+\operatorname{Re} \sum_{j=1}^{\nu} \sum_{k=1}^{d} a_{j k} z_{j} s_{k}+O(|s|(|s|+|t|))
$$

If, say, $\sigma_{1} \neq 0$, we may replace the functions $w_{k}$ by the functions

$$
\begin{array}{r}
\tilde{w}_{1}:=w_{1}-\iota \sum_{i, j=1}^{\nu} \frac{\partial^{2} \phi_{1}}{\partial z_{i} \partial z_{j}}(0) z_{i} z_{j}-\iota \sigma_{1}^{-1} \sum_{j=1}^{\nu} \sum_{k=1}^{d} a_{j k} z_{j} w_{k}, \\
\tilde{w}_{k}:=w_{k}-\iota \sum_{i, j=1}^{\nu} \frac{\partial^{2} \phi_{k}}{\partial z_{i} \partial z_{j}}(0) z_{i} z_{j}, \quad k=2, \ldots, d,
\end{array}
$$

so that, setting $\tilde{s}_{k}:=\operatorname{Re} \tilde{w}_{k}=s_{k}+O(|z|(|z|+|s|+|t|))$ and $\tilde{\Phi}:=\sum_{k=1}^{d} \sigma_{k} \operatorname{Im} \tilde{w}_{k}$, we obtain (after deleting the tildes)

$$
\Phi=\sum_{j=1}^{p^{\prime \prime}}\left|z_{j}\right|^{2}-\sum_{j=p^{\prime \prime}+1}^{p^{\prime \prime}+q^{\prime \prime}}\left|z_{j}\right|^{2}+\frac{1}{2} \sum_{l=1}^{p^{\prime}} t_{l}^{2}-\frac{1}{2} \sum_{l=p^{\prime}+1}^{n-\nu} t_{l}^{2}+O\left(|s|(|s|+|t|)+|z|^{3}+|t|^{3}\right)
$$

We now present an a priori estimate which expresses a necessary condition for local solvability (Lemma VIII.1.1 of Treves [22]). It is a generalization of the classical estimate of Hörmander [11] and Andreotti, Fredricks, and Nacinovich [1].

Let us denote by $\|\cdot\|_{K, l}$, with $K \subset M$ compact and $l \in \mathbb{Z}_{+}$, the seminorms which define the topology of $C^{\infty}\left(M, \Lambda^{p, q}\right)$. Let $m=\operatorname{dim} M-n$.

Lemma 2.2. Let $1 \leq q \leq n$ and $\Omega^{\prime} \subset \Omega \subset M$ be two open neighborhoods such that for every cocycle $f \in C^{\infty}\left(\Omega, \Lambda^{0, q}\right)$ there exists a distribution section $u \in \mathcal{D}^{\prime}\left(\Omega^{\prime}, \Lambda^{0, q-1}\right)$ solving $\mathrm{d}^{\prime} u=f$ in $\Omega^{\prime}$.

Then for every compact subset $K^{\prime} \subset \Omega^{\prime}$ there exist a compact $K \subset \Omega$ and constants $C>0, l \in \mathbb{Z}_{+}$such that, for every cocycle $f \in C^{\infty}\left(\Omega, \Lambda^{0, q}\right)$ and every $v \in C_{c}^{\infty}\left(\Omega^{\prime}, \Lambda^{m, n-q}\right)$, with $\operatorname{supp} v \subset K^{\prime}$ we have

$$
\left|\int_{\Omega} f \wedge v\right| \leq C\|f\|_{K, l}\left\|\mathrm{~d}^{\prime} v\right\|_{K^{\prime}, l}
$$




\section{Proof of Theorem 1.1}

First of all we observe that if the conclusion holds for a given Riemannian metric $\mathbf{g}$, then it holds for every other metric $\mathbf{g}^{\prime}$, since there exist constants $C_{1}>0, C_{2}>0$, $r_{0}>0$ such that

$$
\mathbf{d}\left(x_{0}, x\right) \leq C_{1} \mathbf{d}^{\prime}\left(x_{0}, x\right) \leq C_{2} \mathbf{d}\left(x_{0}, x\right), \quad \forall x \in M \text { with } \mathbf{d}\left(x_{0}, x\right)<r_{0},
$$

where $\mathbf{d}$ and $\mathbf{d}^{\prime}$ are the corresponding distances.

We work in a small neighborhood $\mathcal{O}$ of the point $x_{0}$, that will be taken as the origin of the coordinates, i.e. $x_{0}=0$. There we can make use of the special coordinates introduced in the previous section. Hence $\omega_{0}=\left.\sum_{k=1}^{d} \sigma_{k} d s_{k}\right|_{0}$. We next consider the function $\Phi=\sum_{k=1}^{d} \sigma_{k} \phi_{k}$, which has the form in (2.8). We may assume, without loss of generality, that $\sigma=(1,0, \ldots, 0)$. Consequently, from (2.8) (after the change of variables $t \mapsto t / \sqrt{2}$ ) we have

$$
\phi_{1}(x, y, s, t)=\left|z^{\prime}\right|^{2}-\left|z^{\prime \prime}\right|^{2}+\left|t^{\prime}\right|^{2}-\left|t^{\prime \prime}\right|^{2}+O\left(|s|(|s|+|t|)+|z|^{3}+|t|^{3}\right),
$$

where we set

$$
\left\{\begin{array}{l}
z^{\prime}=\left(z_{1}, \ldots, z_{p^{\prime \prime}}\right), \\
z^{\prime \prime}=\left(z_{p^{\prime \prime}+1}, \ldots, z_{p^{\prime \prime}+q^{\prime \prime}}\right), \\
z^{\prime \prime \prime}=\left(z_{p^{\prime \prime}+q^{\prime \prime}+1}, \ldots, z_{\nu}\right), \\
t^{\prime}=\left(t_{1}, \ldots, t_{p^{\prime}}\right) \\
t^{\prime \prime}=\left(t_{p^{\prime}+1}, \ldots, t_{n-\nu}\right) .
\end{array}\right.
$$

Then, we introduce the Riemannian metric induced by the Euclidean one by means of the coordinates in Proposition 2.1. Hence

$$
B(0, r)=\left\{|z|^{2}+|s|^{2}+|t|^{2}<r^{2}\right\} .
$$

Moreover, we choose a function $\chi \in C^{\infty}\left(\mathbb{R}^{2 \nu+d+(n-\nu)}\right)$, with $\chi=1$ in $B(0,1 / 2)$ and $\chi=0$ away from $B(0,2 / 3)$. We set, for $\delta>0$,

$$
\chi_{\delta, r}(x, y, s, t)=\chi\left(x /\left(\delta r^{3 / 2}\right), y /\left(\delta r^{3 / 2}\right), s /\left(\delta r^{3 / 2}\right), t /\left(\delta r^{3 / 2}\right)\right),
$$

and, for $\rho>0$,

$$
\begin{aligned}
& f_{\rho, \lambda}=e^{\rho h_{1, \lambda}} d \bar{z}^{\prime} \wedge d t^{\prime} \\
& v_{\rho, \lambda}=\rho^{(m+n) / 2} \chi_{\delta, r} e^{\rho h_{2, \lambda}} d \bar{z}^{\prime \prime} \wedge d \bar{z}^{\prime \prime \prime} \wedge d t^{\prime \prime} \wedge d z \wedge d w
\end{aligned}
$$

where, with $\lambda>1$,

$$
h_{1, \lambda}:=-\iota s_{1}+\phi_{1}-2\left|z^{\prime}\right|^{2}-2\left|t^{\prime}\right|^{2}-\lambda \sum_{k=1}^{d}\left(s_{k}+i \phi_{k}\right)^{2},
$$

and

$$
h_{2, \lambda}:=\iota s_{1}-\phi_{1}-2\left|z^{\prime \prime}\right|^{2}-2\left|z^{\prime \prime \prime}\right|^{2}-2\left|t^{\prime \prime}\right|^{2}-\lambda \sum_{k=1}^{d}\left(s_{k}+\iota \phi_{k}\right)^{2} .
$$

We are now going to apply Lemma 2.2 with $f_{\rho, \lambda}$ and $v_{\rho, \lambda}$ in place of $f$ and $v$, respectively, with $\Omega=B(0, r), \Omega^{\prime}=B\left(0, \delta r^{3 / 2}\right)$. Precisely we show that there exist $r_{0}$ and $\delta_{0}$ such that (2.9) fails for every choice of $C$ and $l$ when $\rho \rightarrow+\infty$, if $r<r_{0}$, $\delta>\delta_{0}$, and $\lambda$ is large enough. 
First of all we observe that $f_{\rho, \lambda} \in C^{\infty}\left(\mathcal{O}, \Lambda^{0, q}\right)$ since $p^{\prime \prime}+p^{\prime}=q$ by hypothesis, and $v_{\rho, \lambda} \in C_{0}^{\infty}\left(\mathcal{O}, \Lambda^{m, n-q}\right)$, where $\operatorname{supp} v_{\rho, \lambda} \subset B\left(0, \delta r^{3 / 2}\right)$ is independent of $\rho, \lambda$ (recall $m=\operatorname{dim} M-n$ ). Moreover, by $(2.3)$,

$$
\mathrm{d}^{\prime} f_{\rho, \lambda}=0
$$

and

$$
\mathrm{d}^{\prime} v_{\rho, \lambda}=\rho^{(m+n) / 2} e^{\rho h_{2, \lambda}} \mathrm{d}^{\prime} \chi_{\delta, r} \wedge d \bar{z}^{\prime \prime} \wedge d \bar{z}^{\prime \prime \prime} \wedge d t^{\prime \prime} \wedge d z \wedge d w .
$$

In order to estimate the right-hand side of (2.9) we observe that, by (3.1) and (3.2),

$$
\operatorname{Re} h_{1, \lambda}=-\left|z^{\prime}\right|^{2}-\left|z^{\prime \prime}\right|^{2}-|t|^{2}-\lambda|s|^{2}+\mathcal{R}(s, t)+O\left(|z|^{3}+|t|^{3}\right)+\lambda O\left(|z|^{4}+|t|^{4}\right),
$$

where

$$
|\mathcal{R}(s, t)|=O(|s|(|s|+|t|)) \leq \tilde{C}\left(\frac{\epsilon}{2}(|s|+|t|)^{2}+\frac{1}{2 \epsilon}|s|^{2}\right),
$$

for every $\epsilon>0$. Hence, if $\epsilon$ and then $1 / \lambda$ are small enough, we see that there exist $r_{0}>0$ and $a>0$ such that

$$
\sup _{B(0, r)} \operatorname{Re} h_{1, \lambda} \leq a r^{3}, \quad \forall 0<r<r_{0} .
$$

Similarly,

$$
\begin{aligned}
\operatorname{Re} h_{2, \lambda}=-\left|z^{\prime}\right|^{2}-\left|z^{\prime \prime}\right|^{2}-2\left|z^{\prime \prime \prime}\right|^{2}- & |t|^{2}-\lambda|s|^{2} \\
& +\mathcal{R}^{\prime}(s, t)+O\left(|z|^{3}+|t|^{3}\right)+\lambda O\left(|z|^{4}+|t|^{4}\right),
\end{aligned}
$$

with $\mathcal{R}^{\prime}$ satisfying the same estimate (3.5). Therefore if $\lambda$ is sufficiently large, in $\mathcal{O}$ we have

$$
\operatorname{Re} h_{2, \lambda} \leq-\frac{1}{2}\left(|z|^{2}+|t|^{2}+\lambda|s|^{2}\right)+C_{1}\left(|z|^{3}+|t|^{3}\right)+C_{2} \lambda\left(|z|^{4}+|t|^{4}\right) .
$$

Hence, possibly for a smaller $r_{0}$, there exist constants $b>0, c>0$ such that

$$
\sup _{B(0, r) \backslash B\left(0, \delta r^{3 / 2}\right)} \operatorname{Re} h_{2, \lambda} \leq\left(-b \delta^{2}+c\right) r^{3}, \quad \forall 0<r<r_{0} .
$$

As a consequence of (3.6), (3.7), and (3.4), if $r<r_{0}$, for every compact subset $K \subset B(0, r)$ and any integer $l \geq 0$ it turns out that

$$
\left\|f_{\rho, \lambda}\right\|_{K, l} \leq C^{\prime} \rho^{l} e^{a r^{3} \rho},
$$

and

$$
\left\|\mathrm{d}^{\prime} v_{\rho, \lambda}\right\|_{K, l} \leq C^{\prime \prime} \rho^{(m+n) / 2+l} e^{\left(-b \delta^{2}+c\right) r^{3} \rho},
$$

where the constants $C^{\prime}, C^{\prime \prime}$ depend on $\lambda$ and $r$ but are independent of $\rho$. It follows from (3.8) and (3.9) that if $\delta>\sqrt{(a+c) / b}$ for every $0<r<r_{0}$, it turns out that

$$
\left\|f_{\rho}\right\|_{K, l}\left\|\mathrm{~d}^{\prime} v_{\rho}\right\|_{K, l} \leq C^{\prime} C^{\prime \prime} \rho^{(m+n) / 2+2 l} e^{-\left(b \delta^{2}-a-c\right) r^{3} \rho} \longrightarrow 0, \text { as } \rho \rightarrow+\infty .
$$

We look now at the left-hand side of (2.9). We have

$$
\int f_{\rho, \lambda} \wedge v_{\rho, \lambda}=c \rho^{(m+n) / 2} \int e^{\rho\left(h_{1, \lambda}+h_{2, \lambda}\right)} \chi_{\delta, r} \operatorname{det}\left(\operatorname{Id}_{d \times d}+\iota \frac{\partial \phi}{\partial s}\right) d x d y d s d t
$$

where $0 \neq c \in \mathbb{R}$. Observe that

$$
\left(h_{1, \lambda}+h_{2, \lambda}\right)(x, y, s, t)=-2\left(|z|^{2}+|t|^{2}\right)-2 \lambda|s|^{2}+O\left(|z|^{3}+|s|^{3}+|t|^{3}\right) .
$$


Hence, if $r$ is small enough we have

$$
\operatorname{Re}\left(h_{1, \lambda}+h_{2, \lambda}\right)(x, y, s, t) \leq-\left(|z|^{2}+|t|^{2}+|s|^{2}\right) \quad \text { in } B(0, r) .
$$

We now perform the change of variables $(x, y, s, t) \rightarrow \rho^{-1 / 2}(x, y, s, t)$ in (3.11). Then $\rho \cdot\left(h_{1, \lambda}+h_{2, \lambda}\right)\left(\rho^{-1 / 2} x, \rho^{-1 / 2} y, \rho^{-1 / 2} s, \rho^{-1 / 2} t\right)$ converges pointwise to

$$
-2\left(|z|^{2}+|t|^{2}\right)-2 \lambda|s|^{2}
$$

as $\rho \rightarrow+\infty$. Hence, by virtue of (3.11), (2.1), (3.12) and the Lebesgue convergence theorem, we deduce

$$
\int f_{\rho, \lambda} \wedge v_{\rho, \lambda} \longrightarrow c \int e^{-2\left(|z|^{2}+|t|^{2}\right)-2 \lambda|s|^{2}} d x d y d s d t \neq 0
$$

which, together with (3.10), contradicts (2.9).

This completes the proof of Theorem 1.1.

\section{Some EXAmples}

This example is, in a sense, the model case when $\operatorname{dim} T_{x_{0}}^{0}=1$.

Consider $\mathbb{R}^{2 \nu+1+(n-\nu)}$, with coordinates $x_{j}, y_{j}, s, t_{l}, j=1 \ldots, \nu, l=1, \ldots, n-\nu$, endowed with the locally integrable structure, of the hypersurface type, defined by the first integrals $z_{j}=x_{j}+\iota y_{j}$ and $w=s+\iota \phi(x, y, s, t)$, where

$\phi(x, y, s, t)=\sum_{j=1}^{p^{\prime \prime}}\left|z_{j}\right|^{2}-\sum_{j=p^{\prime \prime}+1}^{p^{\prime \prime}+q^{\prime \prime}}\left|z_{j}\right|^{2}+\sum_{l=1}^{p^{\prime}} t_{l}^{2}-\sum_{l=p^{\prime}+1}^{n-\nu} t_{l}^{2}+O\left(|z|^{3}+|t|^{3}+|s|(|s|+|t|)\right)$, near the origin, with $p^{\prime \prime}+p^{\prime}>0$.

If $p^{\prime \prime}+q^{\prime \prime}=\nu$, then Theorem XVIII.3.1 of [22] applies to any characteristic point of the type $\left(0,\left.\alpha d s\right|_{0}\right), \alpha>0$, above the origin, and yields $\kappa_{q, 0}^{\mathrm{g}}(r)=0$ for every $r<r_{0}, q=p^{\prime}+p^{\prime \prime}$ (and $q=n-p^{\prime}-p^{\prime \prime}$ if $n-p^{\prime}-p^{\prime \prime}>0$ ).

If $p^{\prime \prime}+q^{\prime \prime}<\nu$, then Theorem 1.1 applies to any characteristic point of the type $\left(0,\left.\alpha d s\right|_{0}\right), \alpha>0$, as before, and $\kappa_{q, 0}^{\mathrm{g}}(r)=O\left(r^{3 / 2}\right)$ as $r \searrow 0$, for $q=p^{\prime}+p^{\prime \prime}$ (and $q=q^{\prime \prime}+n-\nu-p^{\prime}$ if $\left.q^{\prime \prime}+n-\nu-p^{\prime}>0\right)$.

We observe that, of course, Theorem 1.1 can be improved in special cases. For example, if $\phi$ in the example above has instead the form

$\phi(x, y, s, t)=\sum_{j=1}^{p^{\prime \prime}}\left|z_{j}\right|^{2}-\sum_{j=p^{\prime \prime}+1}^{p^{\prime \prime}+q^{\prime \prime}}\left|z_{j}\right|^{2}+\sum_{l=1}^{p^{\prime}} t_{l}^{2}-\sum_{l=p^{\prime}+1}^{n-\nu} t_{l}^{2}+O\left(|z|^{4}+|t|^{4}+|s|(|s|+|t|)\right)$,

then an argument similar to the proof of Theorem 1.1 shows that

$$
\kappa_{q, 0}^{\mathrm{g}}(r)=O\left(r^{2}\right), \quad \text { as } r \searrow 0, q=p^{\prime}+p^{\prime \prime} .
$$

\section{ACKNOWLEDGMENTS}

The author wishes to thank P. Cordaro for correspondence on the subject of this paper. The author is also very grateful to M. Nacinovich for providing his paper [10] and to L. Rodino for helpful discussions. 


\section{REFERENCES}

1. A. Andreotti, G. Fredricks, M. Nacinovich, On the absence of the Poincaré lemma in tangential Cauchy-Riemann complexes, Ann. Scuola Norm. Sup. Pisa, Sci. Fis. Mat., 8 (1981), 365-404. MR634855 (83e:32021)

2. A. Andreotti, C.D. Hill, E.E. Levi convexity and the Hans Lewy problem, I and II, Ann. Scuola Norm. Sup. Pisa, Sci. Fis. Mat., 26 (1972), 325-363, 747-806.

3. S. Chanillo, F. Treves, Local exactness in a class of differential complexes, J. Amer. Math. Soc., 10(2) (1997), 393-426. MR1423030 (97m:58185)

4. P.D. Cordaro, J. Hounie, On local solvability of undeterminated systems of vector fields, Amer. J. Math., 112 (1990), 243-270. MR1047299 (91j:58150)

5. P.D. Cordaro, J. Hounie, Local solvability for top degree forms in a class of systems of vector fields, Amer. J. Math., 121 (1999), 487-495. MR1738411 (2001h:58027)

6. P.D. Cordaro, J. Hounie, Local solvability for a class of differential complexes, Acta Math., 187 (2001), 191-212. MR1879848 (2003e:58030)

7. P.D. Cordaro, F. Treves, Homology and cohomology in hypoanalytic structures of the hypersurfaces type, J. Geometric Analysis, 1 (1991), 39-70. MR1097935 (92h:32034)

8. P.D. Cordaro, F. Treves, Hyperfunctions on hypo-analytic manifolds, Annals of Mathematics Studies, 136. Princeton University Press, Princeton, NJ, 1994. MR1311923 (96h:58163)

9. S.-C. Chen, M.-C. Shaw, Partial differential equations in several complex variables, Studies in Advanced Mathematics, Vol. 19, Amer. Mat. Soc., International Press, Providence, RI, 2001. MR1800297 (2001m:32071)

10. C.D. Hill, M. Nacinovich, On the failure of the Poincaré lemma for $\bar{\partial}_{M} I I$, Math. Ann., 335 (2006), 193-219. MR2217688 (2006m:32043)

11. L. Hörmander, Differential operators of principal type, Math. Ann., 140 (1960), 124-146. MR0130574 (24:A434)

12. M. Kashiwara, P. Schapira, A vanishing theorem for a class of systems with simple characteristics, Invent. Math., 82 (1985), 579-592. MR811552 (87g:58120)

13. H. Lewy, An example of a smooth linear partial differential equation without solution, Ann. of Math., 66 (1957), 155-158. MR0088629 (19:551d)

14. V. Michel, Sur la régularité $C^{\infty} d u \bar{\partial}$ au bord d'un domaine de $\mathbb{C}^{n}$ dont la forme de Levi a exactement s valeurs propres négatives, Math. Ann., 295 (1993), 135-161. MR1198845 (93k:32030)

15. M. Nacinovich, Poincaré lemma for tangential Cauchy-Riemann complexes, Math. Ann., 268 (1984), 449-471. MR753407 (86e:32025)

16. M. Nacinovich, On the boundary Hilbert differential complexes, Annales Polonici Mathematici, XLVI (1985), 213-235. MR841829 (88a:58184)

17. M. Nacinovich, On strict Levi q-convexity and q-concavity on domains with piecewise smooth boundaries, Math. Ann., 281 (1988), 459-482. MR954153 (90b:32033)

18. F. Nicola, On the absence of the one-sided Poincaré lemma in Cauchy-Riemann manifolds, Ann. Sci. Norm. Super. Pisa Cl. Sci., (5) 4 (2005), no. 4, 587-600. MR2207735 (2006m:32045)

19. M.M. Peloso, F. Ricci, Tangential Cauchy-Riemann equations on quadratic CR manifolds, Rend. Mat. Acc. Lincei, 13 (2002), 125-134. MR1984107 (2004c:32076)

20. F. Treves, A remark on the Poincaré lemma in analytic complexes with non-degenerate Levi form, Comm. Partial Differential Equations, 7 (1982), 1467-1482. MR679951 (84h:58140)

21. F. Treves, Homotopy formulas in the tangential Cauchy-Riemann complex, Memoirs Amer. Mat. Soc., 87 No. 434 (1990). MR1028234 (90m:32012)

22. F. Treves, Hypo-analytic strucures: Local theory, Princeton University Press, Princeton, 1992. MR1200459 (94e:35014)

23. F. Treves, A treasure trove of geometry and analysis: The hyperquadric, Not. Amer. Mat. Soc., 47 (2000), 1246-1256. MR1784240 (2001h:32056)

Dipartimento di Matematica, Politecnico di Torino, Corso Duca degli Abruzzi 24, 10129 TORINO, ITALY

E-mail address: fabio.nicola@polito.it 